\title{
Polarization conversion by dielectric subwavelength gratings in conical mounting
}

\author{
Nicolas Passilly \\ nicolas.passilly@joensuu.fi \\ Petri Karvinen \\ Kalle Ventola \\ Pasi Laakkonen
}

\section{Jari Turunen}

Jani Tervo

\begin{abstract}
University of Joensuu, Department of Physics and Mathematics, P.O. Box 111, FI-80101 Joensuu, Finland

University of Joensuu, Department of Physics and Mathematics, P.O. Box 111, FI-80101 Joensuu, Finland

University of Joensuu, Department of Physics and Mathematics, P.O. Box 111, FI-80101 Joensuu, Finland

University of Joensuu, Department of Physics and Mathematics, P.O. Box 111, FI-80101 Joensuu, Finland

Nanocomp Ltd, Ensolantie 6, FI-80710 Lehmo, Finland

University of Joensuu, Department of Physics and Mathematics, P.O. Box 111, FI-80101 Joensuu, Finland

University of Joensuu, Department of Physics and Mathematics, P.O. Box 111, FI-80101 Joensuu, Finland
\end{abstract}

Subwavelength dielectric gratings are examined in total-internal-reflection configuration. It is demonstrated experimentally that such elements, fabricated in $\mathrm{TiO}_{2}$, can perform full polarization conversion from incident TE to TM with nearly $100 \%$ efficiency. The dependence of the polarization conversion on the angle of incidence is analyzed. Rigorous diffraction theory is used to cross check the experimental results. [DOI: 10.2971/jeos.2008.08009]

Keywords: Diffraction gratings, polarization, form birefringence

\section{INTRODUCTION}

It is well known that a linear grating with subwavelength periodicity behaves like a slab of birefringent material, namely a negative uniaxial crystal [1]. The first investigations on this class of elements, called form-birefringent gratings, in connection to phase retardation were carried out in 1980's. Transmission-type elements have been designed and fabricated for visible [2]-[6] and infra-red wavelengths [7]-[9]. Most of these papers consider elements that introduce a $\pi / 2$ phase delay between two orthogonal field components, i.e., quarter-wave plates. Retarders with larger phase delay (such as half-wave plates) can be designed similarly, but require a larger groove depth and thus higher aspect ratio (depth/linewidth ratio), which complicates the fabrication task even if high-refractive-index materials are used (see, for example, Ref. [10]).

Qualitatively it is obvious that a reflection-type geometry with oblique incidence, which is appropriate for instance in planar integrated (substrate-mode) optics [11] and flat-panel displays [12]-[14], allows a reduction of groove depth to less than one half of the depth of the corresponding transmission element. Such an approach was taken in Refs. [15] and [16], where total-internal-reflection configuration and a stack of thin films, respectively, were suggested to obtain high reflectivity. Metallic gratings can be used as phase retarders or polarization converters as well, with [17]-[21] or without (explicit) $[15,22,23]$ surface plasmon excitation. The reflec- tivity of most metals is high in the infrared region, where the conductivity is high, but this is not the case in the visible region. We have previously provided theoretical comparisons between metallic and dielectric gratings for polarization conversion in the visible range [24]. Polarization conversion is important in many polarization-manipulating devices such as modulators, integrated switches, amplifiers, polarization splitters and it has also been investigated theoretically using slightly different systems, such as a combination of anisotropic materials and corrugated surfaces [25, 26], a dielectric-film waveguide with a corrugated cover layer [27], or a multilayered structure involving alternating birefringent biaxial layers [28]. Nevertheless, with these techniques, perfect polarization conversion is difficult to achieve and the fabrication seems to be a demanding task.

We have already shown [24] that full conversion with $100 \%$ efficiency is theoretically possible in a certain total-internalreflection configuration (with one conical reflection) with a simple binary dielectric grating. Here we investigate the issue further and provide experimental verification of the previous theoretical predictions. The paper is organized as follows. In Section 2 we present the assumed diffraction geometry of the subwavelength diffractive polarization converters and introduce the design. Section 3 details the fabrication processes of the elements, whereas experimental measurements that give evidence of a full conversion are presented in Section 4. Fi- 
nally, the influence of the incident angle is examined in Secion 5 .

\section{GEOMETRY AND DESIGN}

Let us assume a total-internal-reflection configuration illustrated in Figure 1. The grating region II separates two homogeneous dielectric regions $\mathrm{I}\left(\mathrm{SiO}_{2}\right.$ substrate) and III (air) with refractive indices $n_{0}$ and $n_{3}=1$, respectively. The modulated region II consists of a linear subwavelength-period surface relief grating with period $d$ and depth $h$. In the design we assumed a two-level element with alternating refractive indices $n_{1}$ and $n_{2}=1$, in which case it is customary to define the fill factor as $f=c / d$. However, in order to theoretically crosscheck the experiments, the actual profile shape of the gratings, obtained by SEM measurements, is taken into account. In the analyzed geometry, the incident electromagnetic plane wave arrives from the substrate side (region I) at an input angle $\theta$, which exceeds the critical angle of total internal reflection at the interface between media I and III. The conical angle of incidence, i.e., the azimuthal angle between the plane of incidence and the grating vector, is denoted by $\phi$.

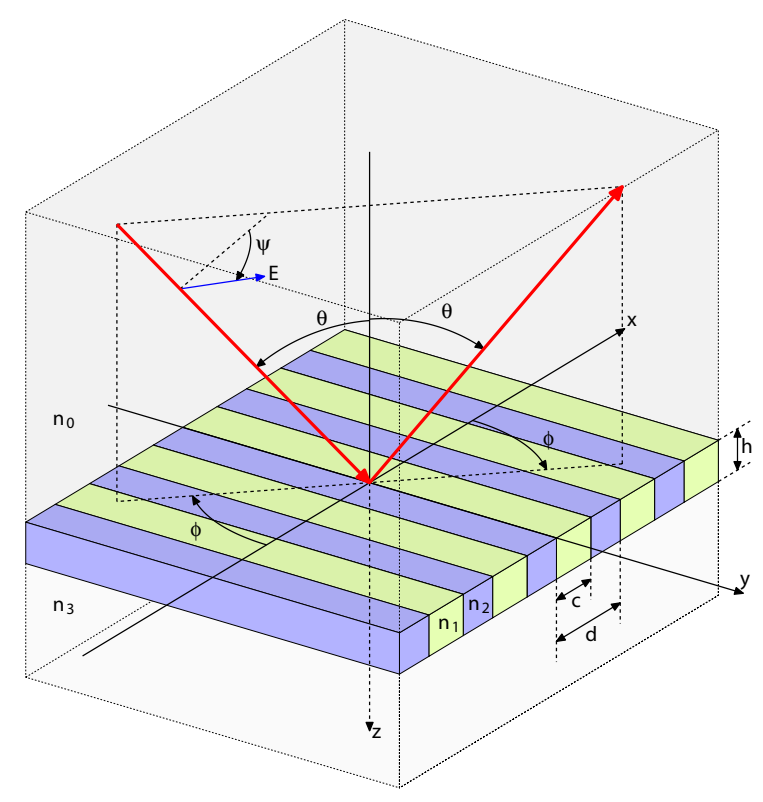

FIG. 1 Diffraction geometry: a plane wave is incident on a binary surface-relief grating from a direction specified by angles $\theta$ and $\phi$, and linearly polarized at an angle $\psi$ with respect to the plane of incidence.

It is well known that for subwavelength gratings below the cut-off period $d_{\text {swl }}$, only the zeroth transmitted and reflected diffraction orders are non-evanescent. This condition, which we shall assume throughout the work, in principle allows high-efficiency polarization conversion provided that an appropriate combination of the angles of incidence and grating parameters can be found to achieve the desired optical function. In the following analysis, we assume that the incident electric field is linearly polarized with an angle $\psi$ with respect to the plane of incidence as illustrated in Figure 1. Phase retardation and polarization conversion for $\psi=45^{\circ}$ have been studied by Haggans et al. [15]. In such a situation, $90^{\circ}$ polarization rotation can be achieved by phase delay only, i.e., without any coupling between the orthogonal electric-field components within the element. In this paper, we focus on a more difficult case of purely TE-polarized incident field with $\psi=90^{\circ}$. Coupling between the field components is then required for polarization conversion and thus the grating must be rotated with respect to the plane of incidence $\left(\phi \neq 0^{\circ}\right.$ or $\left.90^{\circ}\right)$.

Even though subwavelength structures could in principle be analyzed and designed using the effective medium theory, the results provided by such an approach are not sufficiently accurate if the period is close to $d_{\text {swl }}$, mainly because several propagating modes can exist inside the grating layer. Hence we employ the rigorous Fourier Modal Method (FMM) [29] with correct Fourier-factorization rules [30] and stable solution of the boundary conditions [31].

Our design results are summarized in Figure 2, which illustrates the efficiency of the $\mathrm{TE} \rightarrow \mathrm{TM}$ polarization conversion as a function of the $\mathrm{TiO}_{2}$ layer thickness and the conical angle $\phi$ for six different fill factors in subfigures (a)-(f). In the design, we fixed the grating period to $d=250 \mathrm{~nm}$, and the (vacuum) wavelength of the incident TE-polarized field was set to $\lambda=633 \mathrm{~nm}$. Moreover, the assumed refractive indices were $n_{0}=1.46$ for the substrate and $n_{1}=2.08$ for the $\mathrm{TiO}_{2}$ layer. By examining the figure, we see that high polarization conversion is achieved if $f=0.45 \ldots 0.50$ and $h \sim 550 \mathrm{~nm}$. We also note that there exist two "zones" of high polarization conversion: the first zone around $\phi \sim 20^{\circ}$ and the second one around $\phi \sim 60^{\circ}$. The zones are also strongly affected by the fill factor and the thickness: for example, a fill factor $f=0.30$ leads to two zones of high conversion efficiency for roughly the same thickness, while a fill factor $f=0.50$ requires a much shallower structure at $\phi \sim 20^{\circ}$ than at $\phi \sim 60^{\circ}$.

\section{FABRICATION}

To demonstrate the full polarization conversion experimentally, we fabricated two elements with $560 \mathrm{~nm}$ and $460 \mathrm{~nm}$ $\mathrm{TiO}_{2}$-layer thicknesses using standard electron beam lithography [32] and reactive ion etching (RIE). The used substrates were fused silica mask plates (dimensions 5 " $\times 5^{\prime \prime} \times 0.09^{\prime \prime}$ ). Corrugated $\mathrm{TiO}_{2}$ layers have already been utilized for microoptics purpose [33]-[35] but the fabrication technique was based on lift-off. In here, the $\mathrm{TiO}_{2}$ film was evaporated on the substrate, followed by evaporation of a $100 \mathrm{~nm}$ chromium layer. The mask plate was then spin-coated with ZEP-resist (200 nm layer), which was afterwards hard-baked. The electron beam exposure was then performed using a Vistec EBPG $5000+$ ESHR -pattern generator. The resist was developed and used as an etch mask in RIE of the chromium layer in $\mathrm{Cl}_{2} / \mathrm{O}_{2}$ plasma. The remaining resist was removed, and the resulting chromium structure was further used as a mask for the RIE of the $\mathrm{TiO}_{2}$ layer (etching gases $\mathrm{SF}_{6}$ and Ar). In addition to this layer, some overetching of $\mathrm{SiO}_{2}$ also took place. Finally, the residual chromium was removed by wet etching. Scanning electron microscope pictures of the fabricated structures, taken after the measurements, are illustrated in Fig- 

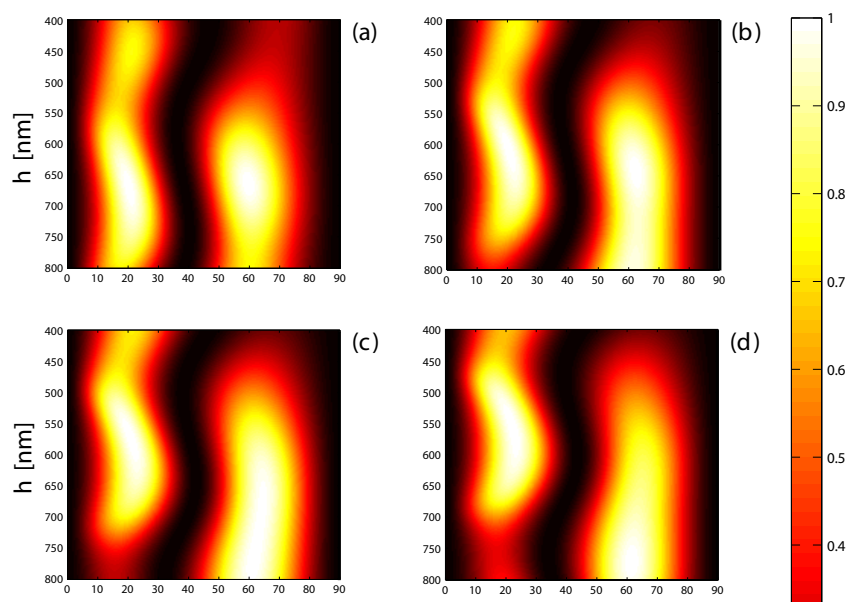

(d)
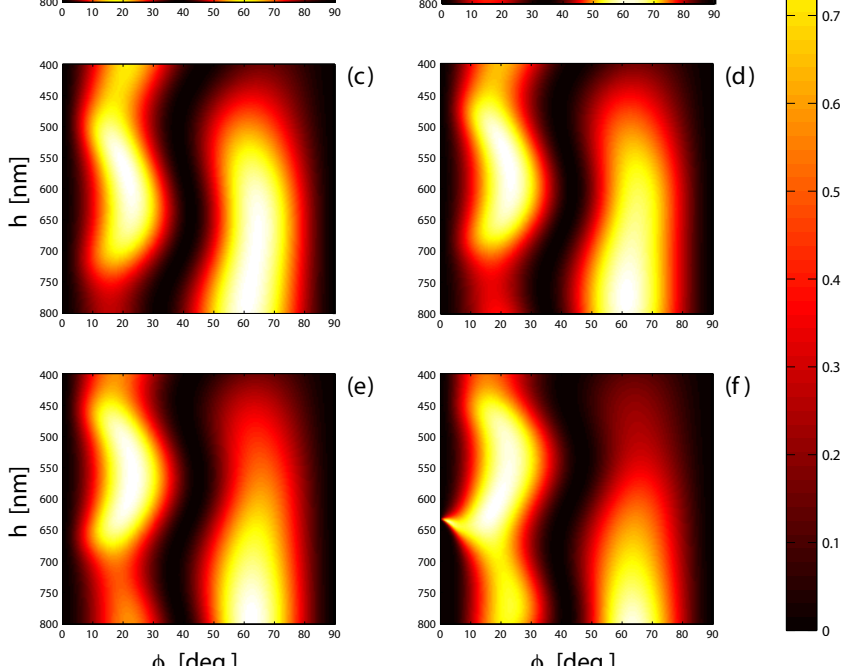

$\phi$ [deg.]

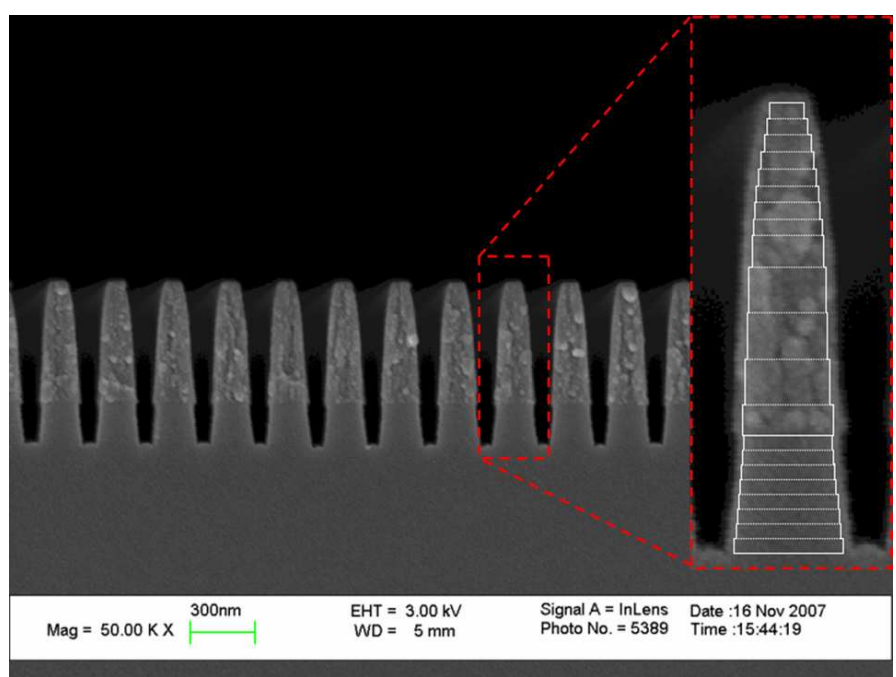

FIG. 3 SEM image of the cross section of the first element with $h=560 \mathrm{~nm} \mathrm{\textrm {TiO } _ { 2 }}$ layer. The right part of the picture shows a magnification of one period, where the shape considered in the cross-checking calculations is drawn. The latter includes a total of 21 layers, 8 for the over-etched part in the substrate, and 13 for the $\mathrm{TiO}_{2}$ part.

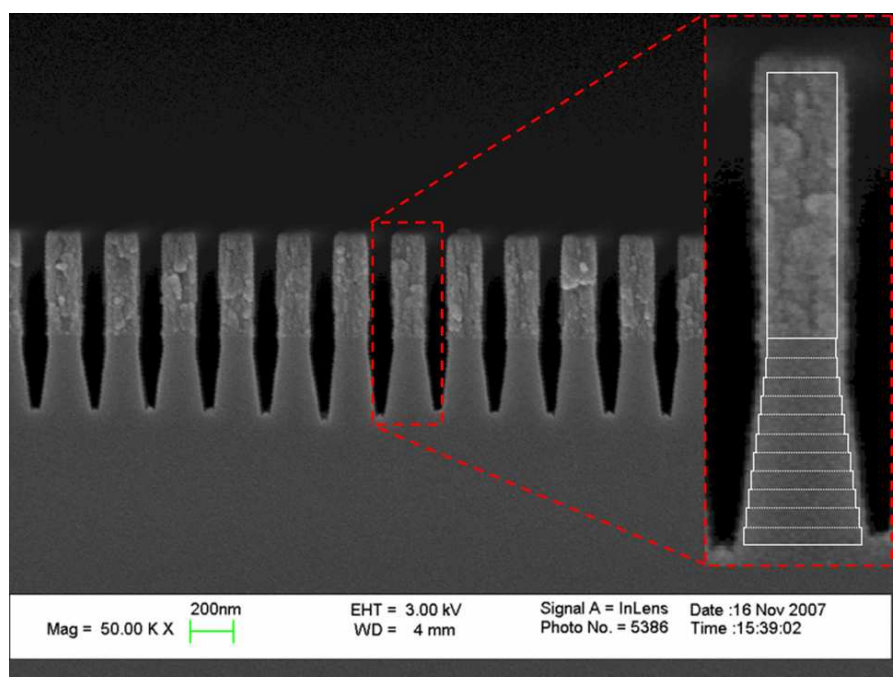

FIC. 4 SEM image of the second element with $h=460 \mathrm{~nm} \mathrm{\textrm {TiO } _ { 2 }}$ layer. The right part now includes a total of 12 layers, 11 for the over-etched part in the substrate and one for the $\mathrm{TiO}_{2}$ part.

equipped with Vernier scales, which allow accurate control of the incident angle.

We began the characterization by setting the incident angle to $45^{\circ}$ using the reflection from the prism. We then measured the zero-order reflected power through the analyzer oriented along TM- or TE-directions. The ratio between the TM and the TE polarized parts and the sum of them (which remains constant for all the conical angles) allows to get normalized efficiencies, denoted by $R_{\mathrm{TM}}$ and $R_{\mathrm{TE}}$. Thus, the precision rotation platform is rotated and experimental values are recorded for different values of $\phi$. We point out that the prism has to be correctly re-oriented for each azimuthal angle so that its faces are always perpendicular to the incident plane and the transmission through the faces of the prism remains the same for the polarization components.

Preliminary measurements were carried out on the first sam- 
ple with a HeNe laser (vacuum wavelength $\lambda=633 \mathrm{~nm}$ ) for an incident angle close to $45^{\circ}$. As mentioned in Section 2, the thickness of the $\mathrm{TiO}_{2}$ layer was measured before the fabrication of the grating and was found to be around $560 \mathrm{~nm}$. For that thickness and a relatively large range of fill factors around $f=0.47$ a binary $\mathrm{TiO}_{2}$ profile shape should lead to a high conversion. However, the first measurements showed that the conversion for the lower values of the azimuthal angle $\left(\phi=21^{\circ}\right)$ was not as high as expected and equal only to $87.3 \%$. The element was behaving more like a thicker element with a thickness closer to $650 \mathrm{~nm}$. This is explained by the overetching of the substrate, which can be seen from the profile of Figure 3. Consequently, the grating behaves as if it was designed for a longer wavelength than $\lambda=633 \mathrm{~nm}$ that was assumed in the original calculations. Indeed, using $\lambda=671 \mathrm{~nm}$ we find that full conversion appears for a binary element with a larger thickness, as illustrated in Figure 5.
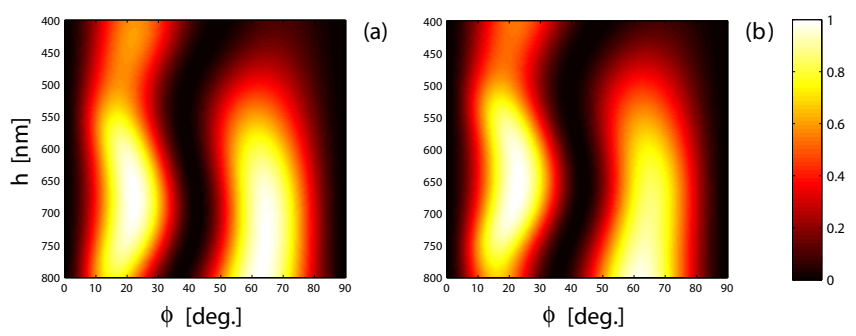

FIG. 5 Calculated conversion efficiencies for binary $\mathrm{TiO}_{2}$ gratings with two different fill factors: (a) $f=0.40$ and (b) $f=0.45$ at $\lambda=671 \mathrm{~nm}$.

Motivated by the results of Figure 5, we tested the first element with a laser-diode-pumped, frequency-doubled $\mathrm{Nd}: \mathrm{YVO}_{4}$ laser at $671 \mathrm{~nm}$. The observed polarization conversion was $99 \%$ at $\phi=16^{\circ}$, as illustrated in Figure 6. Note that at $\phi=0^{\circ}$, no conversion occurs, i.e., $R_{\mathrm{TM}}=0$ and $R_{\mathrm{TE}}=1$ (corresponding to the incident beam) since there is no coupling between the field components.

Figure 6 includes not only experimental points (dots), but also theoretical calculations (solid lines). The theoretical curves are calculated taking into account the actual shape of the grating, obtained from the SEM images in Figure 3. The multi-layer formulation of FMM [29] with 21 layers was employed, 8 of which were used to model the over-etched part inside the substrate and 13 layers to model the $\mathrm{TiO}_{2}$ part. The numerical results are in good agreement with the experimental data. We point out that in order to model the actual shape of the grating, the estimated contribution from the conductive layer to the shape, as visible in the SEM pictures, is not taken into account in the calculations. Moreover, no sizeable scattering has been observed experimentally.

The same measurements were done with the second sample. In this sample, the over-etched part (modeled using 11 layers) allows close to perfect polarization conversion at $\lambda=633 \mathrm{~nm}$ although the nearly binary $\mathrm{TiO}_{2}$ layer itself would be too thin. Measurements were thus performed with a HeNe laser and $94.7 \%$ polarization conversion at $\phi=14^{\circ}$ was observed. The results are summarized in Figure 7. Depending on the align-

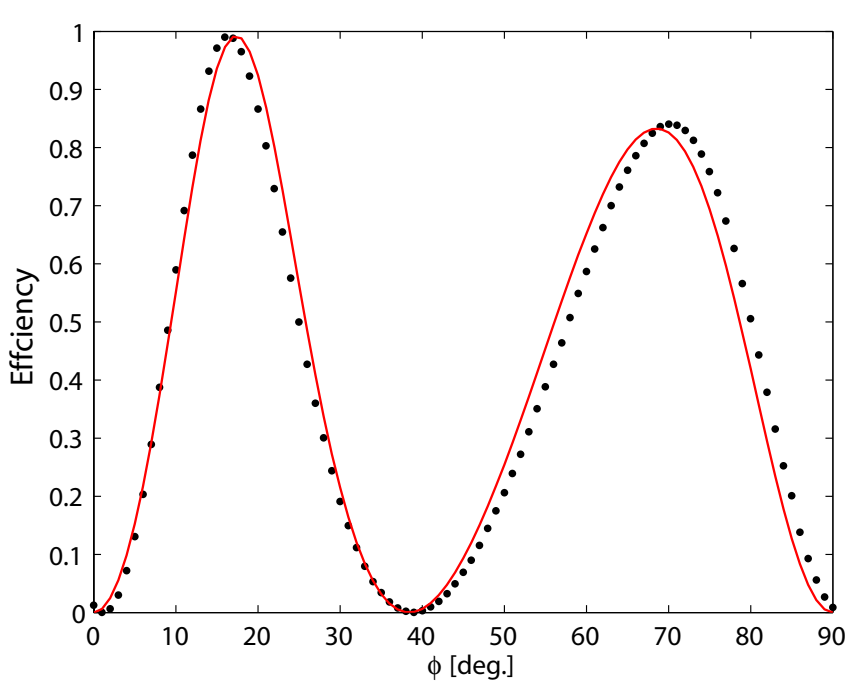

FIG. 6 Polarization conversion by the first element for $\theta=45^{\circ}$ and $\lambda=671 \mathrm{~nm}$. The dots are experimental points and the solid line is plotted from rigorous calculations, which take into account the shape shown in the SEM picture, Figure 3.

ments and positions on the gratings, we can estimate that the error is less than few percents around the high conversion conical positions.

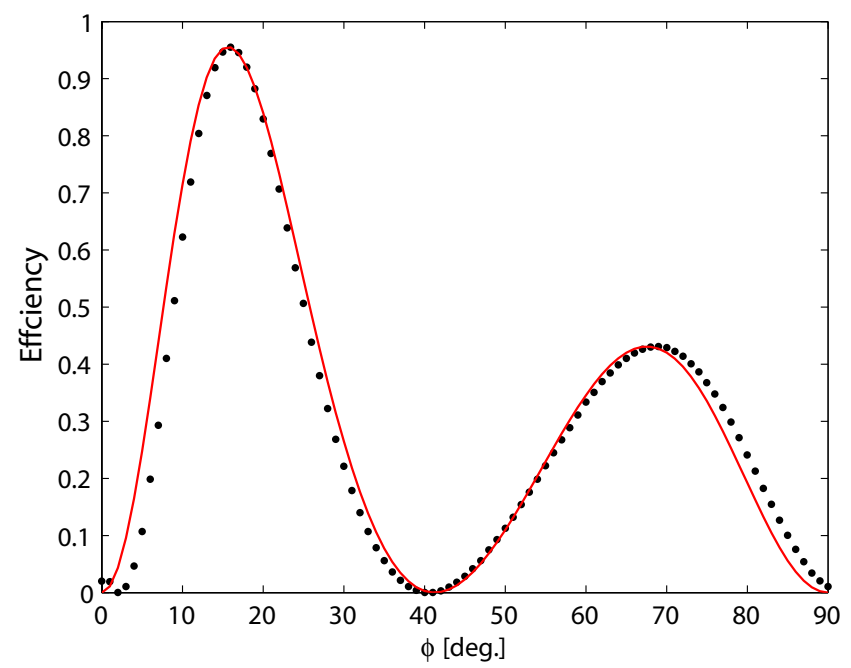

FIG. 7 Polarization conversion by the second element for $\theta=45^{\circ}$ and $\lambda=633 \mathrm{~nm}$. The dots are experimental points and the solid line is a rigorous calculation based on the shape in Figure 4

We remark that the theoretical fitting of the experimental curves is not perfect. This has different reasons: Firstly, there are of course some errors in the various alignments of the setup, which can lead to small (usually expected to be less than few tens of arc. min.) differences of the incident angle and the conical angle compared to the values taken into account in the calculations. Secondly, the fabrication, including resist exposure and development, as well as etching of both the $\mathrm{Cr}$ and the $\mathrm{TiO}_{2}$ layer is not completely uniform. Moreover, when the grating is rotated to record conversion properties at different conical angles, the light usually hits slightly different places at the surface of the element. This should not be a problem in most cases but in the examples analyzed here, 
the polarization conversion is particularly sensitive to some grating parameters. For instance, errors of a few nanometers in the linewidth or layer thickness can lead to significant (several per cent) changes in the conversion efficiency. The calculations are made with one fixed shape and do not take into account possible slight spatial variations. Nevertheless, with further process developments, polarization conversion close to $100 \%$ could be guaranteed.

\section{INFLUENCE OF THE INCIDENT ANGLE}

If we keep in mind that subwavelength gratings behave like artificial uniaxial crystals, albeit the design usually requires rigorous calculations, it is clear that the increase in the optical path leads to larger retardation. This can be achieved by two different ways: Either by increasing the refractive index of the material, as in most of the previous work, or by increasing the propagation length inside the structure. Our total-internalreflection configuration already has the advantage of increasing the propagation length (and hence the optical path) within the grating compared to a transmission-configuration at normal incidence, as shown in previous papers $[24,36]$. Thus it facilitates the required retardation with significantly smaller grating thicknesses. Of course, this propagation length depends on the incident angle, and it is therefore worth testing if we could reduce the required thickness even more by using a higher incident angle than $\theta=45^{\circ}$. Indeed, the incident angle $\theta$ can be a quite free parameter in some applications of substrate mode optics.

In order to check the validity of the above discussion, we changed the angle of the first arm from $44^{\circ}$ to $50^{\circ}$. This corresponds, if the refraction at the prism boundaries is taken into account, to the angles of incidence $\theta=44.34^{\circ}$ to $\theta=48.30^{\circ}$ for $\lambda=671 \mathrm{~nm}$. From Figure 8 we see, as expected, that the increase in $\theta$ greatly affects the polarization conversion. Moreover, if we compare Figures 5 and 8, we find that the increase of $\theta$ has almost the same (qualitative) effect as the increase of $h$, which could also be expected.

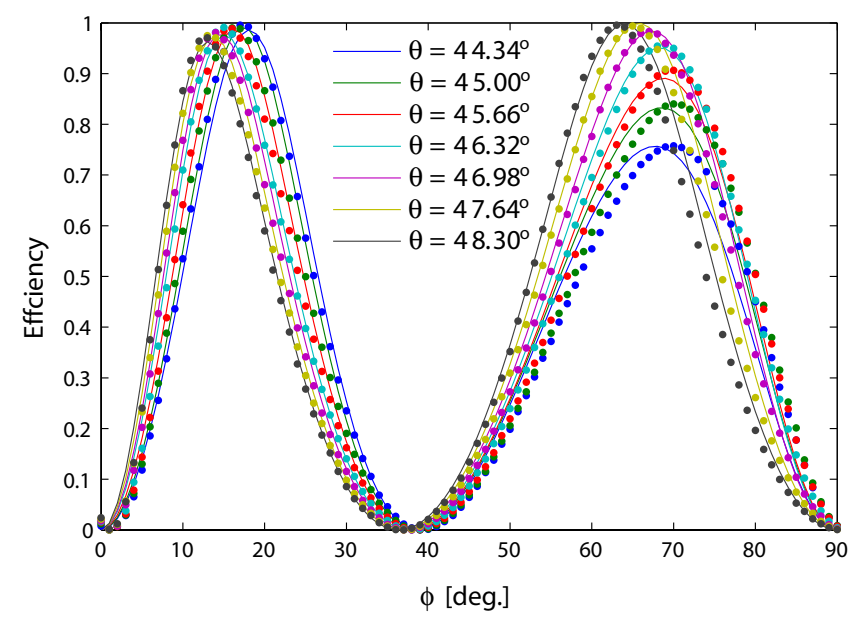

FIG. 8 Polarization conversion by the first element for angles of incidence varying between $44.34^{\circ}$ and $48.30^{\circ}$ and a wavelength $\lambda=671 \mathrm{~nm}$. Dots and solid lines are experimental points and results of rigorous calculation based on Figure 3, respectively.
Examining Figure 8, we find that at conical angles $\phi \sim 20^{\circ}$, the polarization conversion slowly decreases when we increase the angle of incidence. This has its roots in the fact that at $\theta=45^{\circ}$, the element is already slightly thicker than it should be for a full polarization conversion. It is also important to note that under these conditions, a smaller angle of incidence (i.e., $\theta=44.34^{\circ}$ ) tends to increase the conversion, and experimentally $99.5 \%$ conversion has thus been recorded for this angle. On the other hand, the second zone of conversion $\left(\phi \sim 60^{\circ}\right)$ shows a significant increase of the polarization conversion: $99.6 \%$ conversion efficiency was measured for $\phi=62^{\circ}$ and $\theta=48.30^{\circ}$. Again, the comparative numerical results are in good agreement with the experimental data. We note that a very slight fit of the thickness of the $\mathrm{TiO}_{2}$ layer (maximum $5 \mathrm{~nm}$ ) has been made for each curve independently, since the change of the angle of incidence leads to a different active zone of the element. This is absolutely acceptable in view of the fabrication parameters, and helps to get closer to the experimental results.

Similar measurements were made with the second element using illumination with $\lambda=633 \mathrm{~nm}$, and they are shown in Figure 9. For this element, the change in the angle of incidence helps us to increase the polarization conversion for the two zones since, in both cases, the thickness was not large enough to reach the full polarization conversion. For the angle of incidence of $\theta=47.64^{\circ}$, we measured an experimental polarization conversion equal to $97.9 \%$ at $\phi=13^{\circ}$ instead of $94.7 \%$ at $\phi=14^{\circ}$ when $\theta=45^{\circ}$.

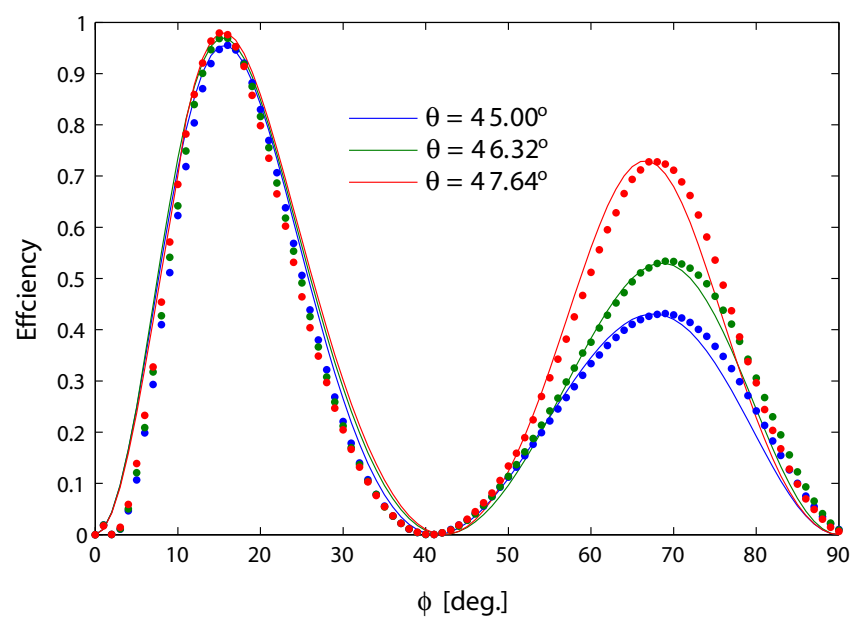

FIG. 9 Polarization conversion by the second element for angles of incidence varying between $45^{\circ}$ and $47.64^{\circ}$ and a wavelength $\lambda=633 \mathrm{~nm}$. Dots and solid lines are experimental points and results of rigorous calculation based on Figure 4 , respectively.

In view of the experimental results, it can be concluded that, as expected, the incident angle can be adjusted to compensate the efficiency of the elements. We can also extrapolate this idea to larger angles of incidence. Figure 10 reports the required thickness $h$ as well as the corresponding conical angle $\phi$ as a function of the fill factor $f$ in order to achieve full polarization conversion $\left(R_{\mathrm{TM}}=1\right)$ for two different angles of incidence $\theta=45^{\circ}$ and $60^{\circ}$. The required thickness, when the incident angle is $\theta=60^{\circ}$, is almost $30 \%$ smaller than the one required for $\theta=45^{\circ}$. Consequently, it can be concluded that 
in case of applications where the incident angle can be a relatively free parameter, it is preferable to use large angles, since in that case full polarization conversion is significantly easier to achieve. This is particularly interesting if production costs and then possibilities of mass-production are taken into account. Indeed, in the case of lower thickness, it would make a technique as Nano-Imprint Lithography (NIL) [37] more successful if one wants to use high refractive index material as $\mathrm{TiO}_{2}$. From another point of view, the help from a longer propagation length could compensate a decrease of the refractive index. Elements made of high-refractive-index UV-moulding materials $(n \simeq 1.7$ ) [12] suitable for mass-production could then be envisaged. For instance, calculations predict that it is possible to reach a full conversion at $\lambda=633 \mathrm{~nm}$ with an element characterized by $d=250 \mathrm{~nm}, h=640 \mathrm{~nm}$, and $f=0.5$, if the refractive index of the layer is $n_{1}=1.7$ and the incident angle is $\theta=60^{\circ}$.

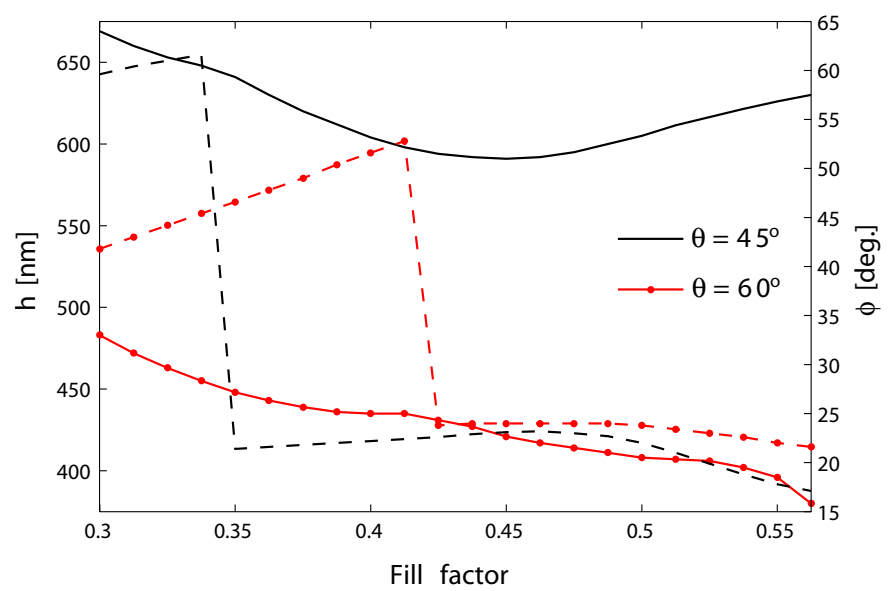

FIG. 10 Required thickness $h$ (solid lines) as a function of the fill factor $f$ to achieve full polarization conversion $\left(R_{\mathrm{TM}}=1\right)$ with the corresponding conical angle $\phi$ (dashed curves). Two angles of incidence are considered: $45^{\circ}$ (black) and $60^{\circ}$ (red).

\section{CONCLUSIONS}

We have presented a study of TE to TM polarization conversion gratings made of dielectric materials that operate in internal reflection mode. Such elements have been fabricated by electron beam lithography and reactive ion etching processes in $\mathrm{TiO}_{2}$ layers and tested experimentally. We showed that a nearly perfect conversion with close to $100 \%$ efficiency is possible for a single wavelength. The influence of the incident angle (to change the equivalent optical path inside the structure) was also investigated in order to look for less-fabricationdemanding possibilities. Good agreement between theory and experiment has been obtained. By extrapolation, we showed that thinner gratings (by tens of per cent) can allow perfect polarization conversion if the incident angle is an adjustable parameter. This could be the case in planar light-guide display applications [12], which is one of the motivations of our work.

\section{ACKNOWLEDGMENTS}

The work was supported in part by the Academy of Finland (projects 111701, 118951, 207523 and 209806). The authors acknowledge the support of the Network of Excellence on Micro-Optics (NEMO), Nokia, Modilis, Nanocomp and the Research and Development Project on Nanophotonics (Ministry of Education, Finland). The funding from the National Agency of Finland (TEKES) is gratefully appreciated.

\section{References}

[1] M. Born and E. Wolf, Principles of Optics, 7th edn. (Cambridge University Press, Cambridge, UK, 1999).

[2] D. C. Flanders, "Submicrometer periodicity gratings as artificial anisotropic dielectrics", Appl. Phys. Lett. 42, 492-494 (1983).

[3] R. C. Enger and S. K. Case, "Optical elements with ultrahigh spatial-frequency surface corrugations", Appl. Optics 22, 32203228 (1983).

[4] L. H. Cescato, E. Gluch, and N. Streibl, "Holographic quarter wave plates", Appl. Optics 29, 3286-3290 (1990).

[5] W. Yu, K. Satoh, H. Kikuta, T. Konishi, and T. Yotsuya, "Synthesis of wave plates using multilayered subwavelength structure", Jpn. J. Appl. Phys. 43, L439-L441 (2004).

[6] T. Claser, S. Schröter, H. Bartelt, H.-J. Fuchs and E.-B. Kley, "Diffractive optical isolator made of high-efficiency dielectric gratings only", Appl. Optics 41, 3558-3566 (2002).

[7] F. Xu, R.-C. Tyan, P.-C. Sun, Y. Fainman, C.-C. Cheng, and A. Scherer, "Fabrication, modeling, and characterization of formbirefringent nanostructures", Opt. Lett. 20, 2457-2459 (1995).

[8] D. L. Brundrett, E. N. Glytsis, and T. K. Gaylord, "Subwavelength transmission grating retarders for use at 10.6 microns", Appl. Optics 35, 6195-6202 (1996).

[9] L. Pang, M. Nezhad, U. Levy, C.-H. Tsai, and Y. Fainman, “Formbirefringence structure fabrication in GaAs by use of SU-8 as a dry-etching mask", Appl. Optics 44, 2377-2381 (2005).

[10] T. Isano, Y. Kaneda, N. Iwakami, K. Ishizuka, and N. Susuki, "Fabrication of half-wave plates with subwavelength structures", Jpn. J. Appl. Phys. 43, 5294-5296 (2004).

[11] J. Jahns, "Planar integration of free-space optical interconnects", P. IEEE 82, 1623-1631 (1994).

[12] T. Levola and P. Laakkonen, "Replicated slanted gratings with a high refractive index material for in and outcoupling of light", Opt. Express 15, 2067-2074 (2007).

[13] K.-W. Chien and H.-P. D. Shieh, "Design and fabrication of an integrated polarized light guide for liquid-crystal-display illumination", Appl. Opt. 43, 1830-1834 (2004).

[14] T. Levola, "Method and optical system for coupling light into a waveguide", U.S. Patent 2005/0002611, Jan.6, 2005.

[15] C. W. Haggans, L. Li, T. Fujita, and R. K. Kostuk, "Lamellar gratings as polarization components for specularly reflected beams", J. Mod. Optic. 40, 675-686 (1993).

[16] V. Kettunen and F. Wyrowski, "Reflection-mode phase retardation by dielectric gratings", Opt. Commun. 158, 41-44 (1998).

[17] G. P. Bryan-Brown, J. R. Sambles, and M. C. Hutley, "Polarisation conversion through the excitation of surface plasmons on a metallic grating", J. Mod. Optic. 37, 1227-1232 (1990). 
[18] S. J. Elston, G. P. Bryan-Brown, T. W. Preist, and J. R. Sambles, "Surface resonance polarization conversion mediated by broken surface symmetry", Phys. Rev. B 44, 3483-3485 (1991).

[19] S. J. Elston, G. P. Bryan-Brown, and J. R. Sambles, "Polarization conversion from diffraction gratings", Phys. Rev. B 44, 6393-6400 (1991).

[20] I. R. Hooper and J. R. Sambles, "Broadband polarization-converting mirror for the visible region of the spectrum", Opt. Lett. 27, 21522154 (2002).

[21] B. T. Hallam, C. R. Lawrence, I. R. Hooper, and J. R. Sambles, "Broad-band polarization convertion from a finite periodic structure in the microwave regime", Appl. Phys. Lett. 84, 849-851 (2004).

[22] Y.-L. Kok and N. C. Gallagher, Jr., "Relative phases of electromagnetic waves diffracted by a perfectly conducting rectangulargrooved grating", J. Opt. Soc. Am. A 40, 65-73 (1988).

[23] R. A. Watts and J. R. Sambles, "Reflection grating as polarization converters", Opt. Commun. 140, 179-183 (1997).

[24] N. Passilly, K. Ventola, P. Karvinen, P. Laakkonen, J. Turunen, and J. Tervo, "Polarization conversion in conical diffraction by metallic and dielectric subwavelength gratings", Appl. Optics 46, 4258-4265 (2007).

[25] R. A. Depine and M. L. Gigli, "Conversion between polarization states at the sinusoidal boundary of a uniaxial crystal", Phys. Rev. B 49, 8437-8445 (1994).

[26] R. E. Inchaussandague and R. A. Depine, "Polarization conversion from diffraction gratings made of uniaxial crystals", Phys. Rev. E 54, 2899-2911 (1996).

[27] S. R. Seshadri, "Polarization conversion by reflection in a thin-film grating", J. Opt. Soc. Am. A 18, 1765-1776 (2001).
[28] N. Ouchani, D. Bria, B. Djafari-Rouhani, and A. Nougaoui, "Transverse-electric/transverse-magnetic polarization converter using 1D finite biaxial photonic crystal", J. Opt. Soc. Am. A 24, 2710-2718 (2007).

[29] L. Li, "A modal analysis of lamellar diffraction gratings in conical mountings", J. Mod. Optic. 40, 553-573 (1993).

[30] L. Li, "Use of Fourier series in the analysis of discontinuous periodic structures", J. Opt. Soc. Am. A 13, 1870-1876 (1996).

[31] L. Li, "Note on the S-matrix propagation algorithm", J. Opt. Soc. Am. A 20, 655-660 (2003).

[32] M. A. McCord and M. J. Rooks, "Electron beam lithography", in Microlithography, P. Rai-Choudhury, ed. (SPIE Press, Bellingham, WA, 1997), Handbook of Microlithography, Micromachining, and Microfabrication, vol. 1, chap. 2.

[33] S. Astilean, P. Lalanne, P. Chavel, E. Cambril and H. Launois, "Highefficiency subwavelength diffractive element patterned in a highrefractive-index material for $633 \mathrm{~nm}$ ", 0pt. Lett. 23, 552-554 (1998).

[34] P. Lalanne, S. Astilean, P. Chavel, E. Cambril and H. Launois, "Design and fabrication of blazed binary diffractive elements with sampling periods smaller than the structural cutoff", J. Opt. Soc. Am. A 16, 1143-1156 (1999).

[35] P. Lalanne, J. Hazart, P. Chavel, E. Cambril and H. Launois, “A transmission polarizing beam splitter grating", J. Opt. A: Pure Appl. Opt. 1, 215-219 (1999).

[36] N. Passilly, K. Ventola, P. Karvinen, J. Turunen, and J. Tervo, "Achromatic phase retardation by subwavelength gratings in total internal reflection", J. Opt. A: Pure Appl. 0pt. 10, 015001 (2008).

[37] J. L. Guo, "Nanoimprint lithography : Methods and material requirements", Adv. Mater. 19, 495-513 (2007). 